Rel at $i$ ons bet ween the $i$ oni zat $i$ on or reconbi nat $i$ on $\mathrm{fl} u \mathrm{ux}$ and the emi ssi on radi at $\mathrm{i}$ on for hydrogen and hel i um i n pl asma

\begin{tabular}{|l|l|}
\hline $\begin{array}{l}\text { jour nal or } \\
\text { publ i cat i on } \mathrm{titl} \text { e }\end{array}$ & Physi cs of Pl asmas \\
\hline vol une & Vol .9 \\
\hline number & I ssue 10 \\
\hline page range & pp. 4316-4324 \\
\hline year & 2002- $10-01$ \\
\hline URL & ht t p: //hdl . handl e. net $/ 10655 / 2219$ \\
\hline
\end{tabular}




\title{
Relations between the ionization or recombination flux and the emission radiation for hydrogen and helium in plasma
}

\author{
Motoshi Goto \\ National Institute for Fusion Science, Toki 509-5292, Japan \\ Keiji Sawada \\ Department of Applied Physics, Faculty of Engineering, Shinshu University, Nagano 380-8553, Japan \\ Takashi Fujimoto \\ Department of Engineering Physics and Mechanics, Graduate School of Engineering, Kyoto University, \\ Kyoto 606-8501, Japan
}

(Received 7 June 2002; accepted 22 July 2002)

\begin{abstract}
On the basis of the collisional-radiative models for neutral hydrogen, and neutral and ionized helium, the relationship between the ionization flux or the recombination flux and the photon emission rate of a representative visible line of each species is investigated. It is found that both fluxes are proportional to the photon emission rate and that the proportionality factor depends rather weakly on the plasma parameters in the ranges of practical interest. This implies that the observed emission line intensity can be a good measure of the ionization flux or the recombination flux. The relation between the total radiation power rate and the ionization or recombination flux is also considered. For a hydrogen plasma in ionization balance the Balmer- $\alpha$ line intensity takes the maximum value near the optimum temperature of $1.3 \mathrm{eV}$, while for plasmas out of ionization balance it takes the minimum near that temperature. This latter characteristic corresponds to the recently observed "inverse edge-localized mode" in divertor plasmas. For neutral hydrogen and ionized helium, it is found that in the recombining plasma of low electron temperature, $T_{e}$, and density, $n_{e}$, the radiation energy close to the ionization potential of the ground state is emitted during one recombination event. In the ionizing plasma of high $T_{e}$ and low $n_{e}$, a similar amount of energy is emitted during one ionization event. Emission line intensities of hydrogen and helium were measured in the Large Helical Device, and the time variation of $n_{e}$ at the initial and final phases of a discharge was estimated. The results agreed well with the interferometer measurement, and this indicated that the variation of $n_{e}$ was dominated by their ionization or recombination processes rather than by diffusion. The total radiation energy of hydrogen and helium in the recombining phase was found to be less than $1 \%$ of the stored energy of the plasma. (C) 2002 American Institute of Physics. [DOI: 10.1063/1.1507588]
\end{abstract}

\section{INTRODUCTION}

In fusion studies the importance of understanding the atomic processes and radiation properties of neutral atoms and low-ionization stage ions in the plasma edge region is increasing. For the detached plasma in the divertor region that is suitable for a steady state operation, the basic mechanisms that trigger the detachment are being intensively investigated. ${ }^{1,2}$ Besides the divertor plasmas, the neutral atom density in the plasma edge region is considered as a key parameter for the transition to the improved confinement mode. ${ }^{3-5}$ In this context it is necessary to elucidate the particle and energy balances in the boundary region where the atomic processes play important roles.

Under such circumstances we consider the relation between the emission line intensity and the effective ionization or recombination rate. Their ratio is useful for the evaluation of particle balances in plasma from the observed emission line intensities. ${ }^{2,6}$ We present here the calculation results for neutral hydrogen and neutral and ionized helium in the wide plasma parameter ranges for various plasma conditions.
These atoms and ions are dominant constituents of fusion plasmas and play important roles in the plasma edge region.

Section II describes the idea of the collisional-radiative (CR) model for neutral hydrogen taken as an example. In Sec. III the relationship between the Balmer- $\alpha$ line intensity and the ionization or recombination flux is discussed. A similar relationship but with the total radiation power is considered in Sec. IV. Section V presents similar quantities to the preceding two sections but for neutral and ionized helium. Finally, in Sec. VI the calculation results are examined in the analysis of the experimental data in the Large Helical Device (LHD).

In this paper we omit the effect of molecular hydrogen. However, it is known that when the molecule is abundant, the emission line intensities of atomic hydrogen are heavily influenced by the excited atoms that are produced in the dissociation processes of molecules. ${ }^{7}$ Therefore, in the analysis of actual divertor plasmas that are characterized by the low temperature and high density parameter regimes, our further understanding of molecular processes and the quan- 


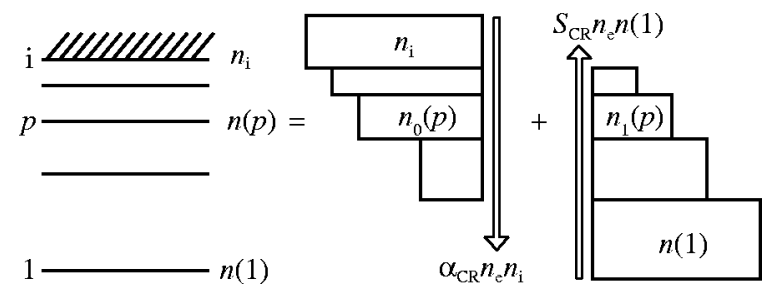

FIG. 1. An illustration of the population structure of excited levels. Each level population $n(p)$ consists of two components, one of which is proportional to the ground state density $n(1)$ and the other is to the ion density $n_{i}$. The ionization and recombination fluxes are also proportional to $n(1)$ and $n_{i}$, respectively.

titative evaluation of the amount of molecules would be necessary.

In the following calculations, we use the CR-model codes developed by the authors of the present paper for neutral hydrogen ${ }^{8}$ and helium. ${ }^{9,10}$ For ionized helium the COLRAD code developed by Rutherford Appleton Laboratory ${ }^{11,12}$ is used.

\section{COLLISIONAL-RADIATIVE MODEL}

We take atomic hydrogen for the purpose of illustration. Let $p$ or $q$ be the principal quantum number of the ground state or an excited level. In plasma the time derivative of the population density of level $p, n(p)$, is expressed as

$$
\begin{aligned}
\frac{d n(p)}{d t}= & -\left\{\sum_{q \neq p} C(p, q) n_{e}+\sum_{q<p} A(p, q)\right. \\
& \left.+S(p) n_{e}\right\} n(p)+\sum_{q \neq p}\left\{C(q, p) n_{e}\right. \\
& +A(q, p)\} n(q)+\left\{\alpha(p) n_{e}+\beta(p)\right\} n_{e} n_{i},
\end{aligned}
$$

where $n_{e}$ and $n_{i}$ are the electron density and the proton density, respectively, $A(p, q)$ is the spontaneous transition probability between level $p$ and $q, C(p, q)$ is the excitation or de-excitation rate coefficient by electron impacts from level $p$ to $q, S(p)$ and $\alpha(p)$ are the ionization rate coefficient by electron impacts and the three-body recombination rate coefficient for level $p$, respectively, and $\beta(p)$ is the radiative recombination rate coefficient for level $p$.

In many cases the relaxation time of excited level populations is short enough as compared with the time constants for ionization and recombination, so that the time derivative in Eq. (1) can be approximated to zero. The validity condition of this approximation is discussed in Ref. 13. Now the coupled equations for all the excited levels are easily solved, and population density $n(p)$ is expressed as a sum of two terms: a term proportional to $n_{i}$ and another to $n(1)$;

$$
n(p)=n_{0}(p)+n_{1}(p)=R_{0}(p) n_{e} n_{i}+R_{1}(p) n_{e} n(1) .
$$

The coefficients $R_{0}(p)$ and $R_{1}(p)$ are functions of the electron temperature, $T_{e}$, and $n_{e}$, and are called the population coefficients. The first and second terms on the right-hand side are called the recombining- and ionizing-plasma components, respectively. These relations are illustrated in Fig. 1.

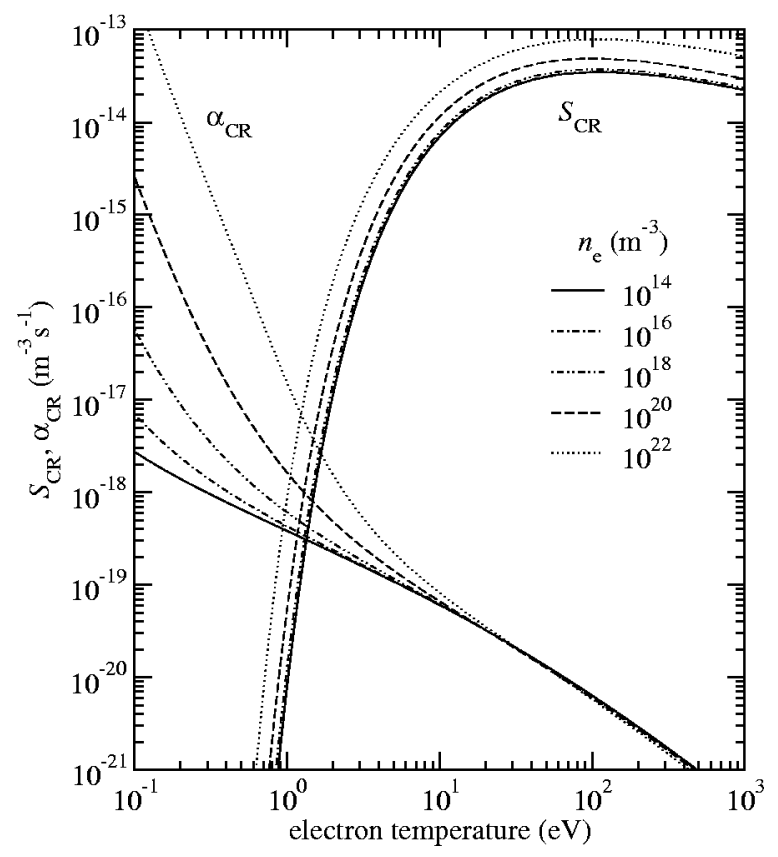

FIG. 2. $T_{e}$ dependences of $S_{\mathrm{CR}}$ and $\alpha_{\mathrm{CR}}$ for several $n_{e}$ values for neutral hydrogen.

The time derivatives of $n(1)$ and $n_{i}$ are expressed in terms of the effective ionization and recombination rate coefficients as

$$
\frac{d n(1)}{d t}=-\frac{d n_{i}}{d t}=-S_{\mathrm{CR}} n_{e} n(1)+\alpha_{\mathrm{CR}} n_{e} n_{i} .
$$

Here, $S_{\mathrm{CR}}$ and $\alpha_{\mathrm{CR}}$ are called the collisional-radiative ionization and recombination rate coefficients, respectively. Figure 2 shows the $T_{e}$ dependences of $S_{\mathrm{CR}}$ and $\alpha_{\mathrm{CR}}$ for several $n_{e}$ values.

For neutral helium the basic idea is the same and the quasi-steady-state approximation is assumed also for the metastable levels here, although, under certain conditions, these levels should be treated independently. ${ }^{9,10}$ The COLRAD code for ionized helium has an option with which the fine structure levels up to $n=4$, where $n$ is the principal quantum number, are treated separately, but here we do not utilize this option and consider only $n$ levels up to $n=50$.

In the COLRAD code the calculation of the collisional transition rate coefficients is limited in the $T_{e}$ range higher than $4000 Z^{2} \mathrm{~K}$, where $Z$ is the nuclear charge. This limitation is inconvenient for our aims, especially for calculations for the recombining plasmas. For the excitation cross section of hydrogenlike ions, Fischer et al. ${ }^{14}$ have introduced two semiempirical formulas proposed by Van Regemorter ${ }^{15}$ and by Vainshtein, Sobelman, and Yukov (VSY), ${ }^{16,17}$ and have determined the fitting parameters for them. Though the authors of Ref. 14 note that their fitting is good for $Z>5$, the rate coefficients calculated with the VSY formula for $Z=2$ are found to agree well with the R-matrix calculation by Aggarwal et al. ${ }^{18,19}$ that are devoted to helium ions for the levels up to $n=4$. Figure 3 shows the comparison of them. We hence adopted the VSY formula with the fitting parameters of Ref. 14 for all the transitions. We have further im- 


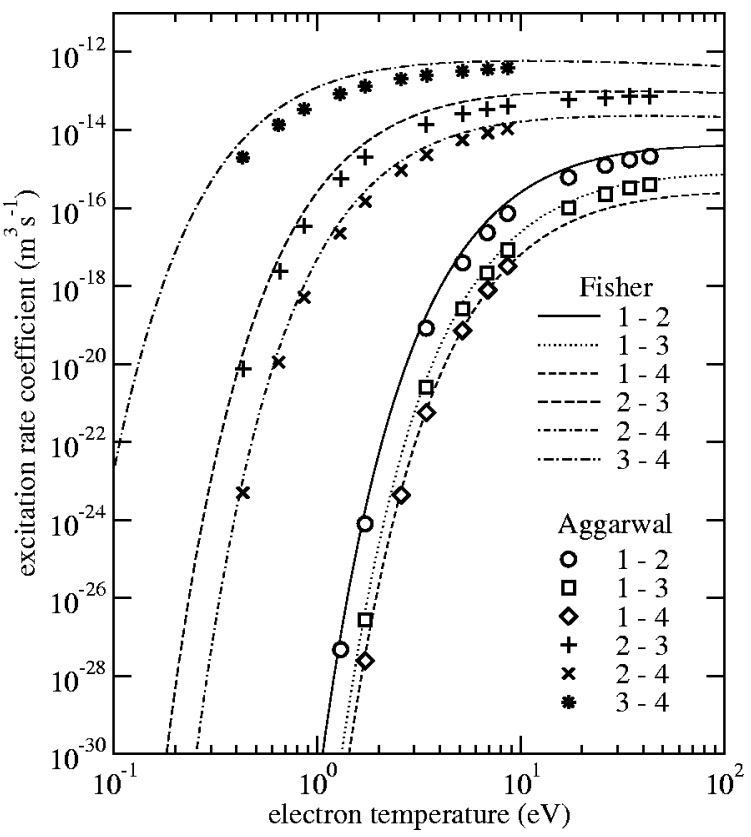

FIG. 3. Comparison of the excitation rate coefficients of ionized helium between the data of Fisher et al. (Ref. 14) and of Aggarwal et al. (Refs. 18 and 19). For the latter the data for the transitions $1-2,1-3$, and 2-3 are taken from Ref. 19, and those for 1-4, 2-4, and 3-4 are from Ref. 18.

proved the radiative recombination rate coefficients by adopting the photoionization cross-section data by Storey and Hummer. ${ }^{20}$

\section{RELATIONS BETWEEN PHOTON EMISSION RATE AND IONIZATION OR RECOMBINATION FLUX}

The first term on the right-hand side of Eq. (3), i.e., the number of ionization events per unit volume per second, is called the "ionization flux," and the second term the "recombination flux." The photon emission rates $A(p, q) n_{0}(p)$ and $A(p, q) n_{1}(p)$ can be rewritten as $\epsilon_{0}(p, q) n_{e} n_{i}$ and $\epsilon_{1}(p, q) n_{e} n(1)$, respectively. Here $\epsilon_{0}(p, q)$ and $\epsilon_{1}(p, q)$ are called the photon emission rate coefficients, and have the same dimension as $S_{\mathrm{CR}}$ and $\alpha_{\mathrm{CR}}$. The ionization flux and the photon emission rate of the ionizing plasma component are both proportional to $n(1)$, and the recombination flux and the photon emission rate of the recombining plasma component are proportional to $n_{i}$. See Fig. 1 .

\section{A. lonizing plasma}

Figure 4(a) shows the $n_{e}$ dependences of $S_{\mathrm{CR}} / \epsilon_{1}(3,2)$ for several $T_{e}$ values. In the $n_{e}$ range lower than $10^{18} \mathrm{~m}^{-3}$ they are almost independent of $n_{e}$. In this range the ionization flux is dominated by the direct ionization from the ground state, i.e.,

$$
S_{\mathrm{CR}} n_{e} n(1) \simeq S(1) n_{e} n(1) .
$$

The population of the level $p=3$ is well approximated by the corona equilibrium as

$$
n_{1}(3) \simeq \frac{C(1,3) n_{e} n(1)}{A(3,1)+A(3,2)},
$$

and hence $\epsilon_{1}(3,2)$ is written as

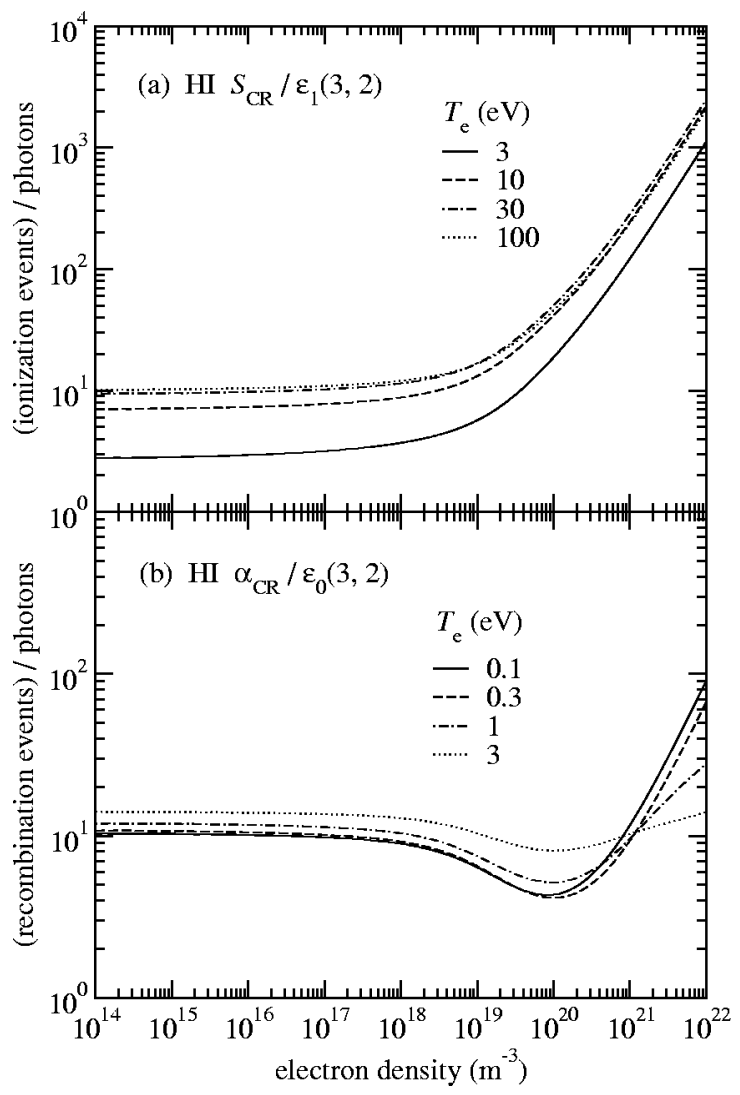

FIG. 4. $n_{e}$ dependences of (a) $S_{\mathrm{CR}} / \epsilon_{1}(3,2)$ and (b) $\alpha_{\mathrm{CR}} / \epsilon_{0}(3,2)$ for several $T_{e}$ values for neutral hydrogen.

$$
\epsilon_{1}(3,2) \simeq \frac{C(1,3) A(3,2)}{A(3,1)+A(3,2)} .
$$

Both $S_{\mathrm{CR}}$ and $\epsilon_{1}(3,2)$ thus show no $n_{e}$ dependences and, as a result, the proportionality factor between them is independent of $n_{e}$.

In the $n_{e}$ range higher than $10^{18} \mathrm{~m}^{-3}, S_{\mathrm{CR}}$ gradually increases with $n_{e}$ due to the additional ionization processes through excited levels, the so-called ladderlike excitationionization process. ${ }^{21}$ Meanwhile, $\epsilon_{1}(3,2)$ steeply decreases with increasing $n_{e}$ due to the change of the population mechanism concerning the level $p=3$; in the influx process the excitation from the $p=2$ level predominates over that from the ground state, and in the outflux process the collisional excitation to the upper levels predominates over the radiative decay. ${ }^{21}$ Thus, $S_{\mathrm{CR}} / \epsilon_{1}(3,2)$ shows a significant $n_{e}$ dependence.

Figure 5 shows the $T_{e}$ dependences of $S_{\mathrm{CR}} n_{e} n(1)$ and $\epsilon_{1}(3,2) n_{e} n(1)$ for $n(1)=1 \mathrm{~m}^{-3}$ under the condition of $n_{e}$ $=10^{18} \mathrm{~m}^{-3}$. It should be noted that, while $S_{\mathrm{CR}} n_{e} n(1)$ increases by six orders when $T_{e}$ is changed from 1 to $10 \mathrm{eV}$, its ratio to $\epsilon_{1}(3,2) n_{e} n(1)$ stays in less than one order, which is consistent with Fig. 4(a).

\section{B. Recombining plasma}

Figure 4(b) shows $\alpha_{\mathrm{CR}} / \epsilon_{0}(3,2)$. It is readily noticed that it depends little on either $n_{e}$ or $T_{e}$ in the low $n_{e}$ range. $\alpha_{\mathrm{CR}}$ can be explicitly written as 


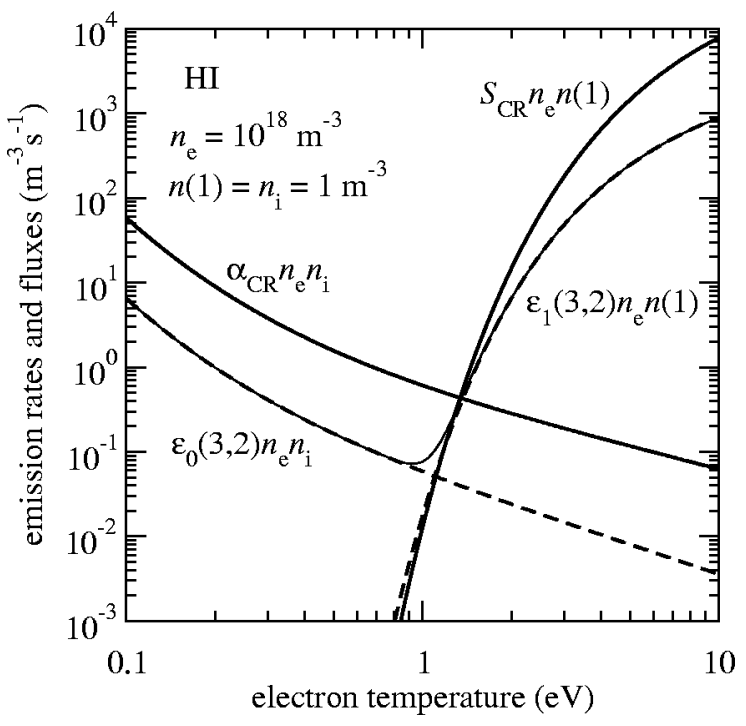

FIG. 5. $T_{e}$ dependences of $\alpha_{\mathrm{CR}} n_{e} n_{i}, S_{\mathrm{CR}} n_{e} n(1), \epsilon_{0}(3,2) n_{e} n_{i}$, and $\epsilon_{1}(3,2) n_{e} n(1)$ under $n(1)=n_{i}=1 \mathrm{~m}^{-3}$ and $n_{e}=10^{18} \mathrm{~m}^{-3}$. Thin solid line indicates the summation of $\epsilon_{0}(3,2) n_{e} n_{i}$ and $\epsilon_{1}(3,2) n_{e} n(1)$.

$$
\alpha_{\mathrm{CR}}=\alpha(1) n_{e}+\beta(1)+\sum_{p}\left\{A(p, 1)+C(p, 1) n_{e}\right\} R_{0}(p) .
$$

Figure 6 shows the $n_{e}$ dependences of the contribution fractions from the various processes to $\alpha_{\mathrm{CR}}$, or the final step to the ground state, for $T_{e}=0.3 \mathrm{eV}$. In the $n_{e}$ range shown here the radiative transitions from the excited levels are the dominant contributions rather than the direct radiative recombination to the ground state $(\beta)$, and this implies that the emission line intensity is closely related to the recombination flux. For other $T_{e}$ values the situation is similar, and the small parameter dependences of $\alpha_{\mathrm{CR}} / \epsilon_{0}(3,2)$ are thus understood. In Fig. 5 the $T_{e}$ dependences of $\alpha_{\mathrm{CR}} n_{e} n_{i}$ and

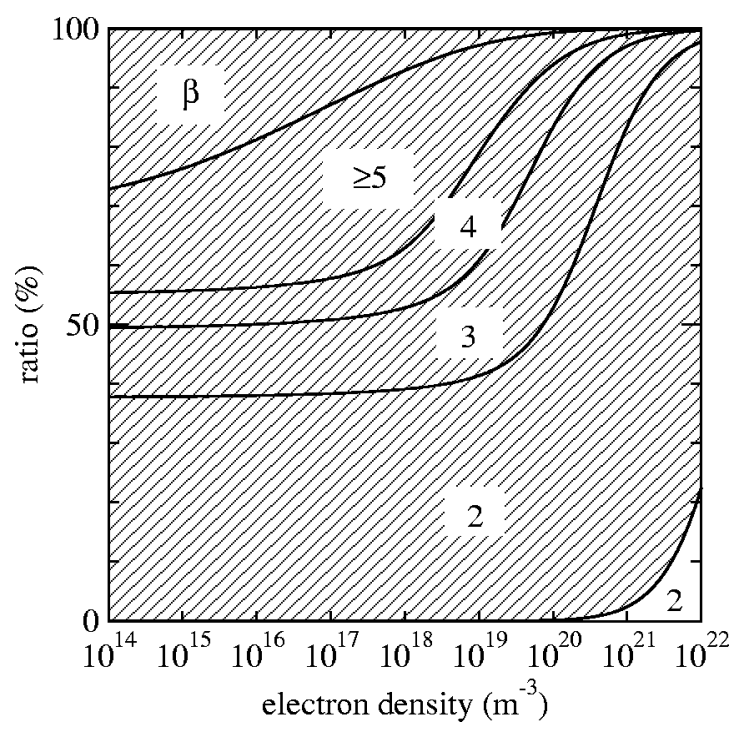

FIG. 6. $n_{e}$ dependences of the contribution fractions from the various processes to $\alpha_{\mathrm{CR}}$. Hatched and not-hatched areas indicate the radiative and collisional processes, respectively. $\beta$ means direct radiative recombination to the ground state.

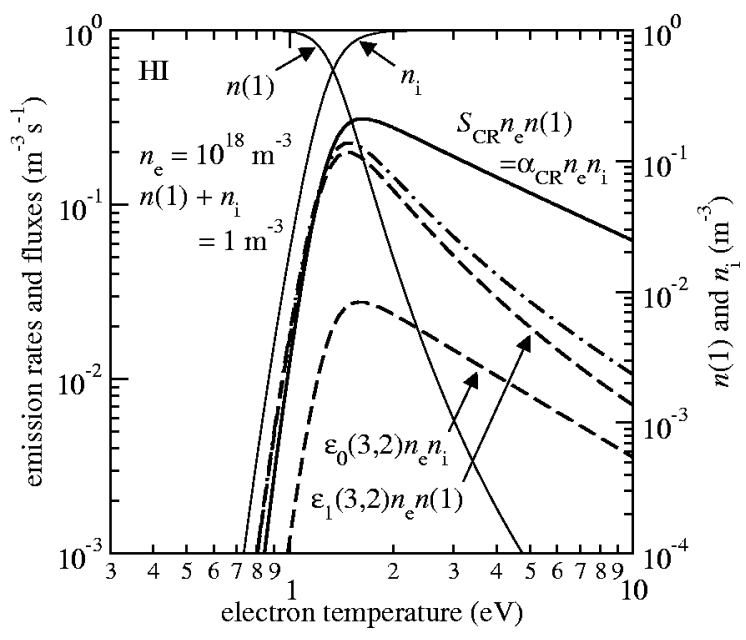

FIG. 7. $T_{e}$ dependences of $n(1)$ and $n_{i}$ under the constraint of $n(1)+n_{i}$ $=1 \mathrm{~m}^{-3}$ for $n_{e}=10^{18} \mathrm{~m}^{-3}$. Also shown $S_{\mathrm{CR}} n_{e} n(1) \quad\left(=\alpha_{\mathrm{CR}} n_{e} n_{i}\right)$, $\epsilon_{0}(3,2) n_{e} n_{i}$, and $\epsilon_{1}(3,2) n_{e} n(1)$ for the same condition. The dash-dotted line is the sum of $\epsilon_{0}(3,2) n_{e} n_{i}$ and $\epsilon_{1}(3,2) n_{e} n(1)$.

$\epsilon_{0}(3,2) n_{e} n_{i}$ for $n_{i}=1 \mathrm{~m}^{-3}$ are also shown and their ratio is found to be almost constant, though each of which changes by about three orders, which is consistent with Fig. 4(b).

\section{Ionization balance plasma}

So far, the ionizing plasma and the recombining plasma have been treated independently. When the ionization flux is balanced with the recombination flux, the ionization balance plasma is established. This situation is realized when the plasma is stationary and homogeneous. In this case the ratio of $n_{i}$ and $n(1)$ is expressed as

$$
\frac{n_{i}}{n(1)}=\frac{S_{\mathrm{CR}}}{\alpha_{\mathrm{CR}}}
$$

Figure 7 shows the $T_{e}$ dependences of $n_{i}$ and $n(1)$ under the constraint of $n(1)+n_{i}=1 \mathrm{~m}^{-3}$ for $n_{e}=10^{18} \mathrm{~m}^{-3}$. The temperature at which $n(1)=n_{i}$ is established $\left(T_{o}\right)$ is about 1.3 eV. Also in Fig. $7 S_{\mathrm{CR}} n_{e} n(1)$ or $\alpha_{\mathrm{CR}} n_{e} n_{i}$, which are identical from the definition, and $\epsilon_{0}(3,2) n_{e} n_{i}$ and $\epsilon_{1}(3,2) n_{e} n(1)$ are shown. The first fact to note is that, in this plasma, these photon emission rates are of similar magnitude especially for higher $T_{e}$. Another is that these rates and their sum take maximum values at around $T_{e}=T_{o}$, and this temperature is hence called the "optimum temperature." Thus, it may be concluded that when an emission line intensity is strong, the temperature of the plasma should be close to $T_{o}$. But we must remember that this statement is correct so long as the plasma is in ionization balance.

In the temperature range higher than $T_{o}$ the decrease in $S_{\mathrm{CR}} n_{e} n(1)$ and $\epsilon_{1}(3,2) n_{e} n(1)$ with rising $T_{e}$ is due to the steeply decreasing $n(1)$. The coefficients $S_{\mathrm{CR}}$ and $\epsilon_{1}(3,2)$ themselves continue to increase with $T_{e}$ in this temperature range (See Figs. 2 and 5). A similar explanation applies to the decrease of $\alpha_{\mathrm{CR}} n_{e} n_{i}$ and $\epsilon_{0}(3,2) n_{e} n_{i}$ in the temperature range lower than $T_{o}$.

Here, we focus on the fact that the temperature where $S_{\mathrm{CR}} n_{e} n(1)$ or $\alpha_{\mathrm{CR}} n_{i} n_{i}$ takes maximum ( $\left.T_{o}^{\prime}\right)$ is slightly 
higher than $T_{o}$ as seen in Fig. 7. In this case, $T_{o}^{\prime}$ is about 1.6 $\mathrm{eV}$, and at this temperature the ratio $n_{i} / n(1)$ is about 9, much larger than 1 at $T_{e}=T_{o}$. This can be understood as follows. The state where $T_{e}=T_{o}^{\prime}$ can be defined as

$$
\frac{d}{d T_{e}}\left[S_{\mathrm{CR}} n(1)\right]=\frac{d}{d T_{e}}\left[\alpha_{\mathrm{CR}} n_{i}\right]=0 .
$$

Under this condition the ratio of $n_{i}$ and $n(1)$ is expressed as

$$
\begin{aligned}
{\left[\frac{n_{i}}{n(1)}\right]_{T_{o}^{\prime}} } & =-\left(\frac{d S_{\mathrm{CR}} / d T_{e}}{S_{\mathrm{CR}}}\right) /\left(\frac{d \alpha_{\mathrm{CR}} / d T_{e}}{\alpha_{\mathrm{CR}}}\right) \\
& =-\left(\frac{d\left(\log S_{\mathrm{CR}}\right)}{d\left(\log T_{e}\right)}\right) /\left(\frac{d\left(\log \alpha_{\mathrm{CR}}\right)}{d\left(\log T_{e}\right)}\right) .
\end{aligned}
$$

From Fig. 2 the derivatives $d\left(\log S_{\mathrm{CR}}\right) / d\left(\log T_{e}\right)$ and $d\left(\log \alpha_{\mathrm{CR}}\right) / d\left(\log T_{e}\right)$ are estimated to be about 9 and -1 , respectively, at $T_{e}=1.6 \mathrm{eV}$ for $n_{e}=10^{18} \mathrm{~m}^{-3}$. As a result, $n_{i} / n(1) \sim 9$ is obtained and this agrees with the result in Fig. 7.

\section{Plasmas out of ionization balance}

For real plasmas, the great majority is out of ionization balance by many orders of magnitude. An example is a transient plasma: i.e., $T_{e}$ changes much more rapidly than the ionization/recombination of the plasma itself. ${ }^{22}$ Another example is the plasma in which particle transport plays an important role. For example, the transition from the attached to detached phase of the divertor plasma is understood as a steep decrease in the temperature where the ion flow from the plasma core and the atom flow from the divertor plate remain almost unchanged. In such cases, Fig. 5 represents the situation better than Fig. 7; In Fig. 5 we assume $n(1)$ $=n_{i}=1\left(\mathrm{~m}^{-3}\right)$ and $T_{e}$ varies. For $T_{e}>T_{o}$ our plasma is an ionizing plasma and for $T_{e}<T_{o}$ our plasma is a recombining plasma. For plasmas out of ionization balance, either the ionizing plasma component or the recombining plasma component is dominant. In this sense, this figure is quite universal in that, for $T_{e} \gg T_{o}$ or $T_{e} \ll T_{o}$, only one of the components is dominant and the other is negligibly small. For this particular condition of $n(1)=n_{i}$, the emission line intensity is minimum at around $T_{e}=T_{o}$. This is exactly consistent with the fact that at around this temperature the sum of the ionization flux and the recombination flux takes the minima. The "inverse edge-localized modes phenomena" observed in JET (Joint European Terus) ${ }^{23}$ and ASDEX Upgrade ${ }^{24}$ and the "negative spikes" in the emission line intensity in the transition between the attached and detached states of plasma observed in NAGDIS-II ${ }^{1}$ can be interpreted in the context of the present explanation. It is noted here that these minima correspond exactly to the maxima in the case of the ionization balance plasma in Fig. 7.

\section{TOTAL RADIATION POWER}

In Sec. III we have focused our attention on the relation between the intensity of a single emission line and the ionization or recombination flux. When we consider, for example, the radiation loss in the divertor region with the re-

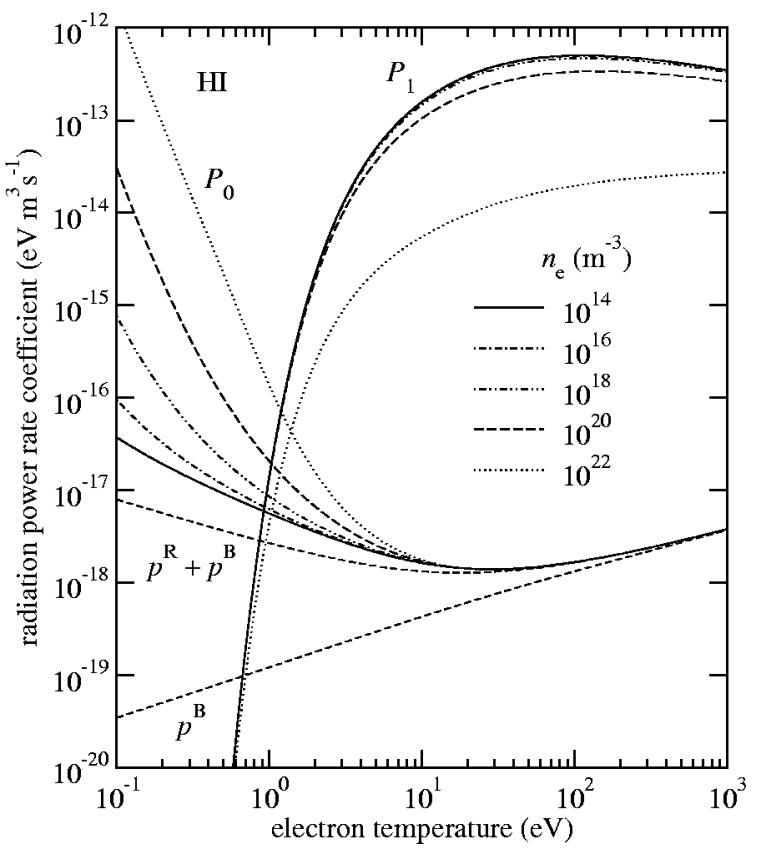

FIG. 8. $T_{e}$ dependences of $P_{0}$ and $P_{1}$ for several $n_{e}$ values for neutral hydrogen.

duction of heat flux on the divertor plates in mind, what should be considered is the total radiation power including all the line emissions and the continuum radiation.

The total radiation power density of neutral hydrogen, $W_{\mathrm{H}}$, is expressed as

$$
\begin{aligned}
W_{\mathrm{H}}= & \sum_{p} n(p)\left(\sum_{q<p} A(p, q) \chi_{q p}\right)+W^{R}+W^{B} \\
= & \sum_{p}\left[R_{0}(p) n_{e} n_{i}+R_{1}(p) n_{e} n(1)\right]\left(\sum_{q<p} A(p, q) \chi_{q p}\right) \\
& +p^{R} n_{e} n_{i}+p^{B} n_{e} n_{i} \\
= & P_{0} n_{e} n_{i}+P_{1} n_{e} n(1)
\end{aligned}
$$

where $\chi_{q p}$ is the transition energy between the levels, $p$ and $q$, and $W^{R}$ and $W^{B}$ are the contributions from the continuum radiation accompanying the radiative recombination processes and the bremsstrahlung, respectively. The coefficients $p^{R}$ and $p^{B}$ in the rewritten forms of $W^{R}$ and $W^{B}$ are the functions of $T_{e}$. We call $P_{0}$ and $P_{1}$ the radiation power rate coefficient of the recombining plasma component and the ionizing plasma component, respectively.

Figure 8 shows the $T_{e}$ dependences of $P_{0}$ and $P_{1}$ for several $n_{e}$ values. Here, the fractions of $p^{R}$ and $p^{B}$ are also shown. The gaunt factors for free-free transitions that are needed in the evaluation of $p^{B}$ are calculated with the code of Ref. 20. For $P_{1}$ it is found that in the low $n_{e}$ cases the $n_{e}$ dependence is weak. This is understood from the fact that when $n_{e}$ is low, the corona equilibrium is valid for most of the excited levels and their population is proportional to $n_{e}$ as mentioned in Sec. III A. The decrease in $P_{1}$ in the high $n_{e}$ cases is due to the change of the population mechanisms of the excited levels as already mentioned in Sec. III A. 


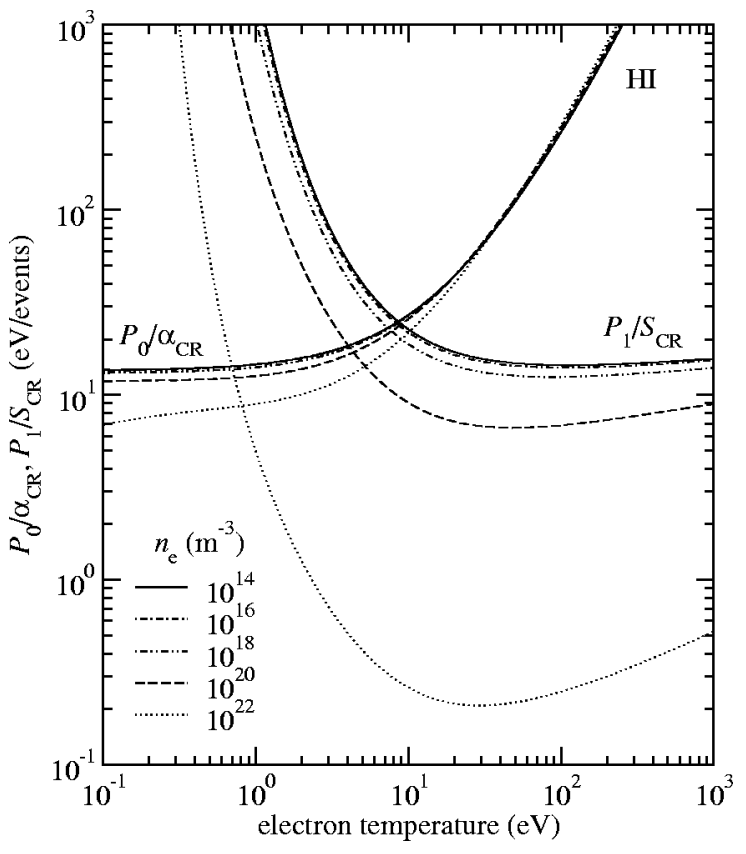

FIG. 9. $T_{e}$ dependences of $P_{0} / \alpha_{\mathrm{CR}}$ and $P_{1} / S_{\mathrm{CR}}$ for several $n_{e}$ values for neutral hydrogen.

Figure 9 shows the ratio $P_{0} / \alpha_{\mathrm{CR}}$. In the low $T_{e}$ range, where the contribution from the bremsstrahlung is small, the values are close to the ionization potential of the ground state atom $(\sim 13.6 \mathrm{eV})$. This is understood as: in this regime the electrons having low kinetic energies are mainly captured to the ions through the radiative recombination processes, and even those which are captured to the excited levels eventually decay to the ground state by cascades. As $n_{e}$ increases the ratio is slightly reduced. This is due to the enhanced role of the collisional processes which replace the radiative decay.

In Fig. 9 the ratio $P_{1} / S_{\mathrm{CR}}$ is also shown. The remarkable feature is that in the low $n_{e}$ and high $T_{e}$ ranges, the ratio is also close to the ionization potential of the ground state atom. This fact is understood from the following consideration. In the corona equilibrium regime virtually all the electrons excited from the ground state eventually return to the ground state by radiative transitions either directly or via other lower excited levels. In such circumstances $P_{1}$ can be approximately written as

$$
\begin{aligned}
P_{1} & \simeq \sum_{p=2}^{\infty} C(1, p) \chi_{1 p} \\
& \simeq \chi_{H} C(1,2) \frac{3^{4}}{2^{5}} \sum_{p=2}^{\infty} \frac{p^{3}}{\left(p^{2}-1\right)^{3}} \\
& =\chi_{H} C(1,2) \frac{3^{4}}{2^{5}}\left[\frac{\zeta(3)}{4}+\frac{3}{32}\right] \\
& \simeq 0.998 \chi_{H} C(1,2),
\end{aligned}
$$

where $\chi_{H}$ and $\zeta(k)$ are the ionization potential of the ground state and the Riemann zeta function, respectively, and the theoretical approximation ${ }^{21}$

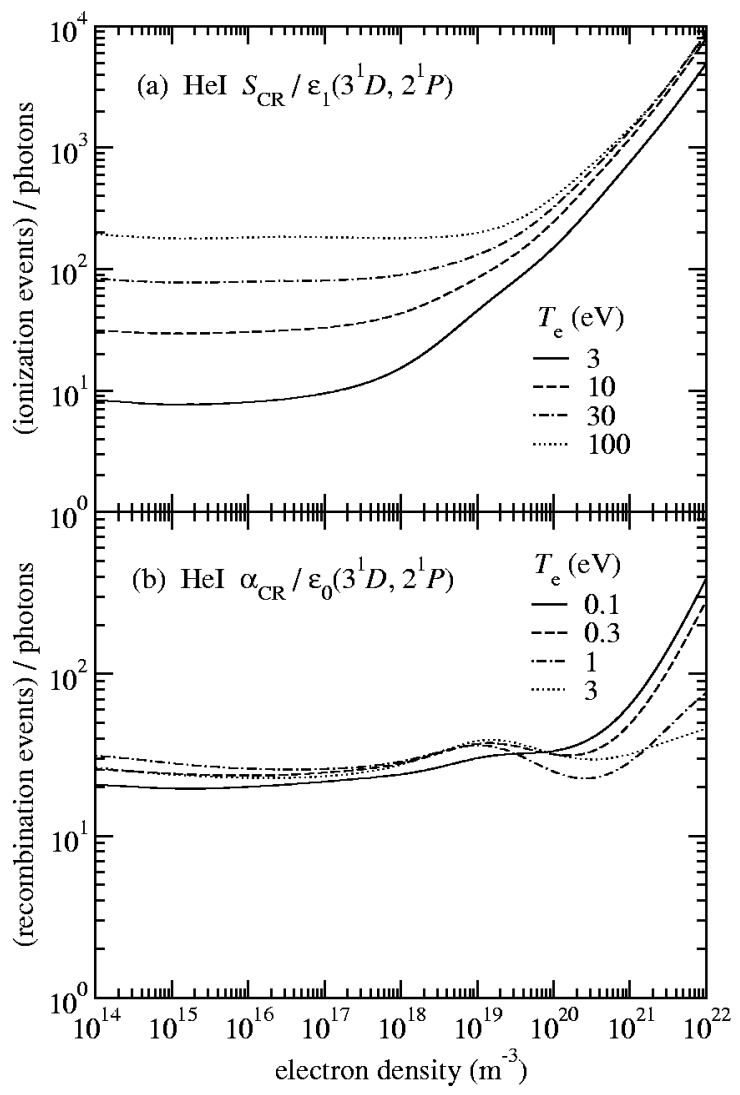

FIG. 10. $n_{e}$ dependences of (a) $S_{\mathrm{CR}} / \epsilon_{1}\left(3{ }^{1} D, 2{ }^{1} P\right)$ and (b) $\alpha_{\mathrm{CR}} / \epsilon_{0}\left(3{ }^{1} D, 2{ }^{1} P\right)$ for several $T_{e}$ values for neutral helium.

$$
C(1, p) \propto \frac{1}{p^{3} \chi_{1 p}^{4}}
$$

is used. As for the rate coefficient $C(1,2)$, the following approximation holds well in the low $n_{e}$ and high $T_{e}$ ranges, ${ }^{21}$

$$
S_{\mathrm{CR}} \simeq S(1) \simeq C(1,2) .
$$

$P_{1}$ is thus found to be close to $\chi_{H} S_{\mathrm{CR}}$. When $T_{e}$ is low, $S_{\mathrm{CR}}$ deviates from $C(1,2)$, and when $n_{e}$ increases, collisional processes become significant, and then the relation $P_{1}$ $\simeq \chi_{H} S_{\mathrm{CR}}$ breaks down.

\section{NEUTRAL AND IONIZED HELIUM}

Calculations similar to those in Secs. III and IV have been carried out for neutral and ionized helium. The emission lines, He I $\lambda 667.8 \mathrm{~nm}\left(2{ }^{1} P-3{ }^{1} D\right)$ and He II $\lambda 468.6$ $\mathrm{nm}(3-4)$, are taken as examples. The results $\alpha_{\mathrm{CR}} / \epsilon_{0}(p, q)$ and $S_{\mathrm{CR}} / \epsilon_{1}(p, q)$ for neutral helium are shown in Fig. 10 and those for ionized helium are shown in Fig. 11. Similar features to the case of neutral hydrogen are observed.

Figure 12 shows $P_{0}$ and $P_{1}$ for neutral helium. Here, $P_{0}$ includes the radiation by the stabilizing transition in the process of the dielectronic recombination; This is responsible for the "hump" in $10 \mathrm{eV} \leqslant T_{e} \leqslant 100 \mathrm{eV}$. Figure 13 shows $P_{0} / \alpha_{\mathrm{CR}}$ and $P_{1} / S_{\mathrm{CR}}$. Due to the rather complicated energylevel structure as compared with neutral hydrogen, the values deviate from the ionization potential of the ground state $(\sim 24.59 \mathrm{eV})$ even in the parameter ranges that are favored 


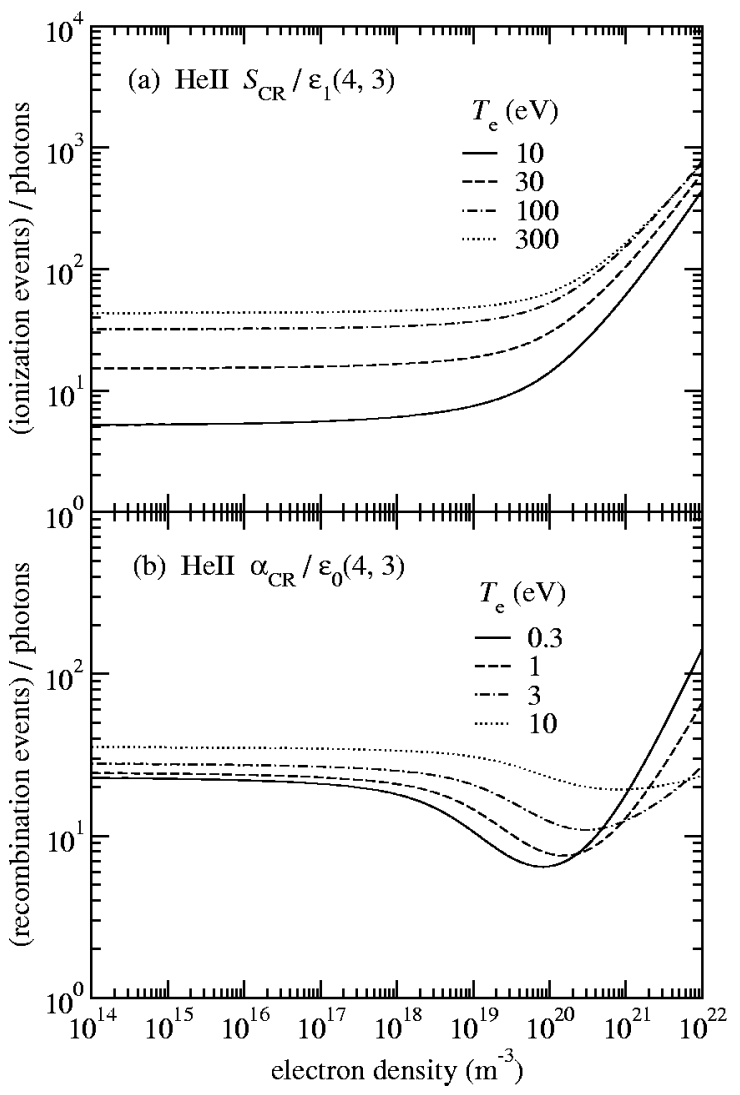

FIG. 11. $n_{e}$ dependences of (a) $S_{\mathrm{CR}} / \epsilon_{1}(4,3)$ and (b) $\alpha_{\mathrm{CR}} / \epsilon_{0}(4,3)$ for several $T_{e}$ values for ionized helium.

for $P_{0} \simeq \chi_{H} \alpha_{\mathrm{CR}}$ and $P_{1} \simeq \chi_{H} S_{\mathrm{CR}}$ for neutral hydrogen. It is, however, noteworthy that they still have similar values in such parameter ranges.

Figure 14 shows $P_{0}$ and $P_{1}$, and Fig. 15 shows $P_{0} / \alpha_{\mathrm{CR}}$

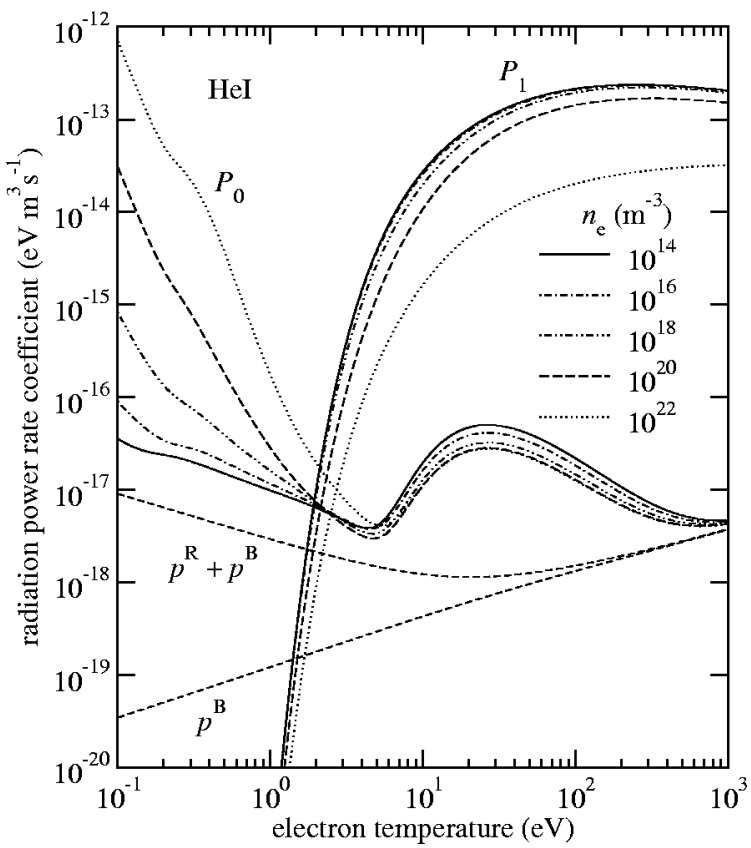

FIG. 12. $T_{e}$ dependences of $P_{0}$ and $P_{1}$ for several $n_{e}$ values for neutral helium.

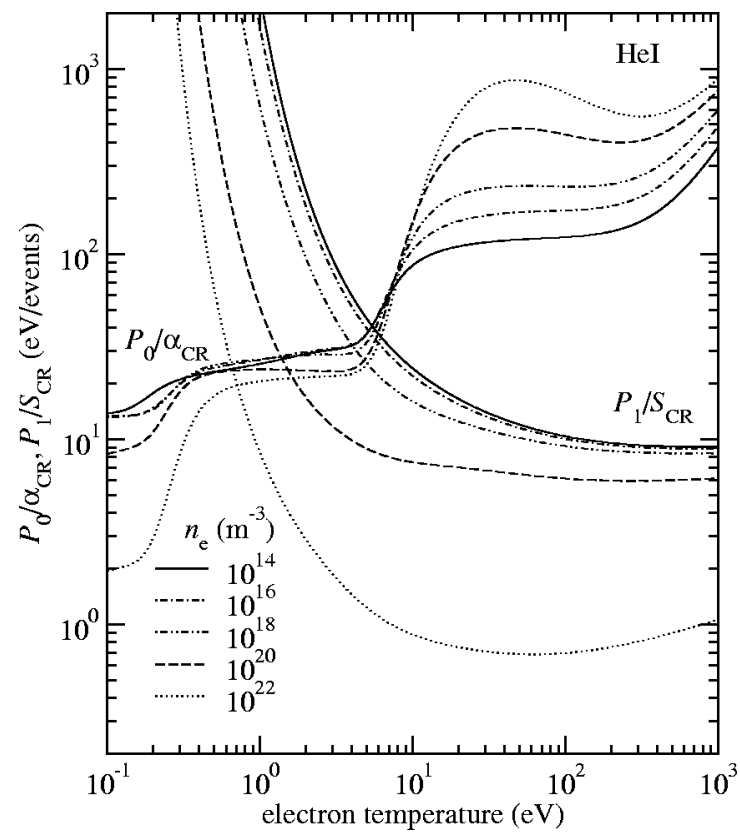

FIG. 13. $T_{e}$ dependences of $P_{0} / \alpha_{\mathrm{CR}}$ and $P_{1} / S_{\mathrm{CR}}$ for several $n_{e}$ values for neutral helium.

and $P_{1} / S_{\mathrm{CR}}$ for ionized helium. It is again found that $P_{0} / \alpha_{\mathrm{CR}}$ in the low $T_{e}$ and low $n_{e}$ ranges and $P_{1} / S_{\mathrm{CR}}$ in the high $T_{e}$ and low $n_{e}$ ranges have similar values to the ionization potential of the ground state ion $(\sim 54.4 \mathrm{eV})$.

It is known that neutral hydrogen and hydrogenlike ions follow the scaling against the nuclear charge. The energy and $T_{e}$ scale according to $Z^{2}$, and $n_{e}$ scales according to $Z^{7} .{ }^{25} \mathrm{It}$ is seen that the results in Figs. 9 and 15 approximately follow these scalings. These scalings make ionized helium interesting from the standpoint of radiation loss. In comparison

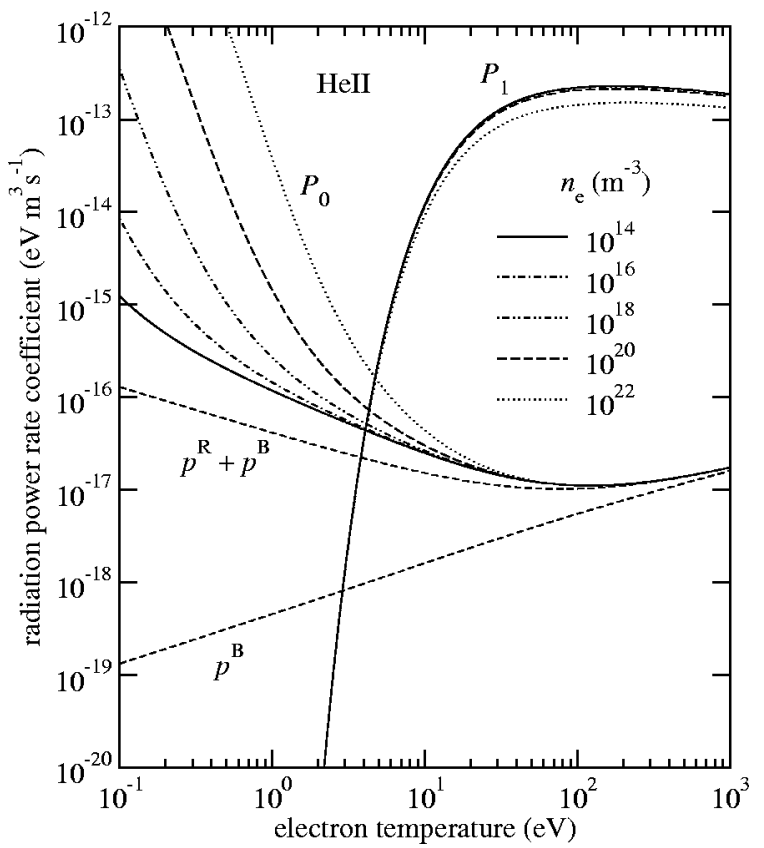

FIG. 14. $T_{e}$ dependences of $P_{0}$ and $P_{1}$ for several $n_{e}$ values for ionized helium. 


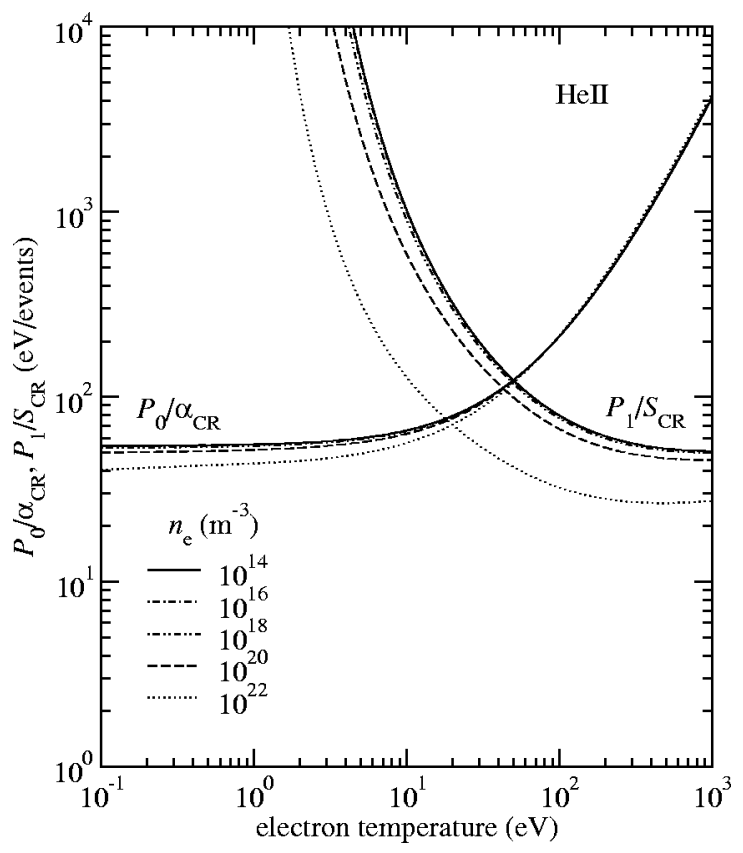

FIG. 15. $T_{e}$ dependences of $P_{0} / \alpha_{\mathrm{CR}}$ and $P_{1} / S_{\mathrm{CR}}$ for several $n_{e}$ values for ionized helium.

with neutral hydrogen, ionized helium tends to radiate more energy per ionization event under plasma conditions of practical interest. For instance, in a plasma with $T_{e}=10 \mathrm{eV}$ and $n_{e}=10^{22} \mathrm{~m}^{-3}$, neutral hydrogen emits $0.3 \mathrm{eV}$ per ionization event while ionized helium emits $100 \mathrm{eV}$. This observation may have some significance in the efforts to reduce the heat flux to divertor plates.

\section{EXAMPLE}

In many real plasmas like those in LHD, at the beginning of a discharge an ionizing plasma is formed and $n_{e}$ increases through the ionization flux. On the other hand, at the end of discharge $n_{e}$ may be diminished by diffusion and the recombination flux in a recombining plasma.

We have measured the intensities of the H I $\lambda 656.3 \mathrm{~nm}$, He I $\lambda 667.8 \mathrm{~nm}$, and He II $\lambda 468.6 \mathrm{~nm}$ lines in a helium discharge. Though only helium gas is puffed, an appreciable amount of hydrogen is found present which is considered to originate from the neutral beam injection (NBI) or from the wall. The viewing chord of the observation almost coincides with the major axis of an elliptical poloidal cross section of the plasma which is elongated in the major radius direction. The measurement has been carried out with a spectrometer equipped with a charge coupled device detector and all three lines are measured simultaneously. For the present measurement the sampling time is $5 \mathrm{~ms}$. Figure 16(a) shows the time evolution of the photon emission rate of the abovementioned three lines at the beginning of a discharge. Since hydrogen and helium are the dominant constituents of the plasma, the following relationship is expected to be a good approximation:

$$
\frac{d}{d t} n_{e}=\kappa_{1} I_{1}+\kappa_{2} I_{2}+\kappa_{3} I_{3},
$$

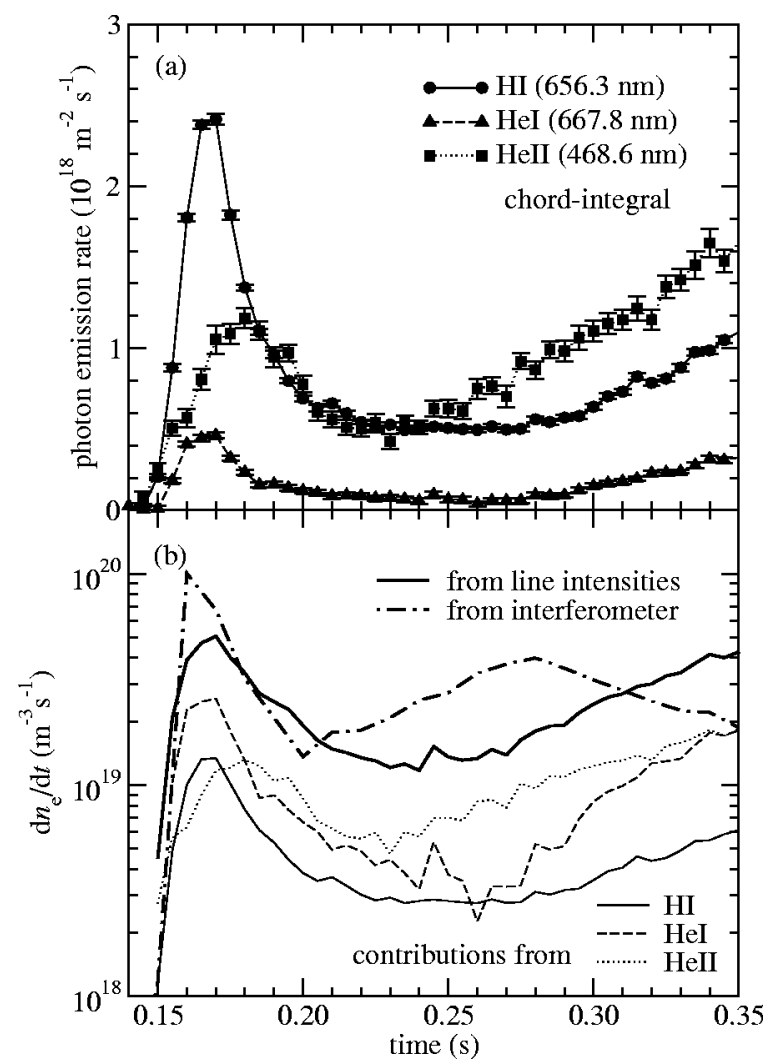

FIG. 16. Time variations of (a) the photon emission rates of the $\mathrm{H}_{\mathrm{I}} \lambda 656.3$ $\mathrm{nm}(n=2-3)$, He I $\lambda 667.8 \mathrm{~nm}\left(2{ }^{1} P-3{ }^{1} D\right)$ and He II $\lambda 468.6 \mathrm{~nm}(n$ $=3-4$ ) lines and (b) the time derivative of $n_{e}$ estimated from the photon emission rates of the above three emission lines and that from the interferometer measurement in the initial stage of a discharge.

where 1, 2, and 3 stand for H I $\lambda 656.3 \mathrm{~nm}$, He I $\lambda 667.8 \mathrm{~nm}$, and He II $\lambda 468.6 \mathrm{~nm}$ lines, respectively, $I$ is the measured photon emission rates, and $\kappa$ corresponds to $S_{\mathrm{CR}} / \epsilon_{1}(p, q)$.

In the time region displayed in Fig. 16, $n_{e}$ is measured with the interferometer for the same discharge and found to be $2 \times 10^{19} \mathrm{~m}^{-3}$ at highest, and $T_{e}$ has been estimated to be in the range of $30-100 \mathrm{eV}$ from the emission line intensity ratios of neutral helium. ${ }^{10}$ Taking these parameters into account, we fix $\kappa_{1}, \kappa_{2}$, and $\kappa_{3}$ to be 10,100 , and 20 , respectively (see Figs. 4, 10, and 11). The right-hand side of Eq. (21) is calculated with these values and the photon emission rates in Fig. 16(a) and the results are shown in Fig. 16(b), together with that deduced from the interferometer measurement. Here, the viewing chord length, $\sim 1.8 \mathrm{~m}$, is used for both the results. It is noted that we have ignored the parameter dependences of $\kappa$, spatial and temporal, and the existence of other impurities and that the toroidal location of our measurement is different from that of the interferometer. Considering such circumstances and that the comparison is conducted in the time derivatives, which are prone to errors, we conclude that the agreement is satisfactory and the validity of the present method is confirmed.

Figure 17(a) shows the photon emission rates of the same emission lines near the end of the same discharge. It is noted that we have subtracted the intensity of the blended He II $(n=4-6)$ line from that of the H I $\lambda 656.3 \mathrm{~nm}$ line. The contribution is estimated from the intensity of the He II $\lambda$ 


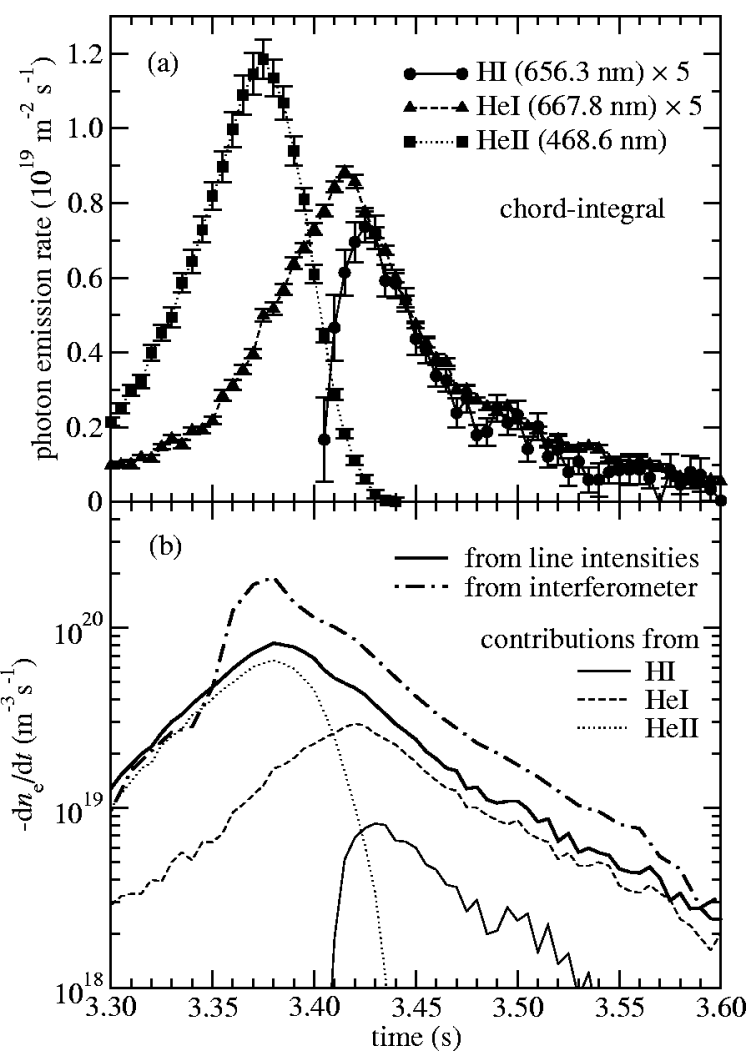

FIG. 17. Similar to Fig. 16, but for the final stage of a discharge.

$273.3 \mathrm{~nm}(n=3-6)$ line measured in a different but similar discharge. Equation (21) is expected to be valid with the negative $\kappa$ which corresponds to $-\alpha_{\mathrm{CR}} / \epsilon_{0}(p, q)$. When the intensity of the He II $\lambda 468.6 \mathrm{~nm}$ line takes the maximum, $n_{e}$ is about $1 \times 10^{19} \mathrm{~m}^{-3}$ and later decreases. $T_{e}$ has been estimated from the population distribution over the excited levels of neutral and ionized helium as compared with the results of CR-model calculations. In both the cases $T_{e}$ is found to be lower than $1 \mathrm{eV}$. Bearing these values in mind, we assume constant values for $\kappa_{1}, \kappa_{2}$, and $\kappa_{3}$ as $-10,-30$, and -10 , respectively (see Figs. 4, 10, and 11). The righthand side of Eq. (21) is calculated and the results are shown in Fig. 17(b). The agreement is again good and the validity of the present method is confirmed also for the recombining phase. This result implies that at the end of a discharge in LHD, $n_{e}$ is diminished by the recombination processes rather than diffusion.

For neutral hydrogen, for example, the quantity $P_{0} / \epsilon_{0}(3,2)$ is estimated to be about $100 \mathrm{eV} /$ photons from Figs. 4 and 9 in the parameter ranges of the recombining phase. For neutral and ionized helium the corresponding quantity is estimated to be about 300 and 1000, respectively. From these values, the measured photon emission rates, and the plasma volume $\left(\sim 30 \mathrm{~m}^{3}\right)$, the total radiation energy from the plasma in the recombining phase is evaluated to be about $30 \mathrm{~J}$ for neutral hydrogen, $140 \mathrm{~J}$ for neutral helium, and $1900 \mathrm{~J}$ for ionized helium. These values are less than $1 \%$ of the total stored energy of the plasma, $300 \mathrm{~kJ}$, just before the termination of NBI heating. After the heating is terminated, most of the stored energy is lost in about $500 \mathrm{~ms}$, while $n_{e}$ is kept almost constant in this time duration, and then the recombination of hydrogen and helium follows. This indicates that though the recombination processes of hydrogen and helium play important roles for the decrease of $n_{e}$ as mentioned previously, they have almost nothing to do with the dominant decrease of the stored energy or the temperature.

We have throughout assumed the quasi-steady-state approximation for the metastable levels, but its validity is questionable in the low density cases. For example, under the condition of $T_{e}=0.1 \mathrm{eV}$ and $n_{e}=10^{16} \mathrm{~m}^{-3}$, the decay time of $2^{1} S$ level to the ground state through the electron collisions is about $20 \mathrm{~ms}$, which is comparable to the decay time of $n_{e}$ in Fig. 17. In such circumstances, however, other processes like metastable-metastable collisions and diffusion of metastable atoms may play important roles in the population balance, and the quasi-steady-state approximation may still be valid. ${ }^{9}$ For more precise analyses of the experiment, such a deliberate calculation would be necessary.

\section{ACKNOWLEDGMENTS}

We wish to acknowledge helpful discussions with Dr. Shigeru Morita. We also thank the entire LHD staff for supporting the experiment.

${ }^{1}$ N. Ohno, D. Nishijima, S. Takamura, Y. Uesugi, M. Motoyama, N. Hattori, H. Arakawa, N. Ezumi, S. Krasheninnikov, A. Pigarov, and U. Wenzel, Nucl. Fusion 41, 1055 (2001).

${ }^{2}$ E. M. Hollman, A. Yu. Pigarov, R. Seraydarian, D. G. Whyte, and S. I. Krasheninnikov, Phys. Plasmas 9, 1226 (2002).

${ }^{3}$ R. L. Boivin, J. A. Goetz, A. E. Hubbard, J. W. Hughes, I. H. Hutchinson, J. H. Irby, B. LaBombard, E. S. Marmar, D. Mossessian, C. S. Pitcher, and J. L. Terry, Phys. Plasmas 7, 1919 (2000).

${ }^{4}$ T. Fülöp, P. J. Catto, and P. Helander, Phys. Plasmas 8, 5214 (2001).

${ }^{5}$ R. J. Groebner, M. A. Mahdavi, A. W. Leonard, T. H. Osborne, G. D. Porter, R. J. Colchin, and L. W. Owen, Phys. Plasmas 9, 2134 (2002).

${ }^{6}$ M. Goto and S. Morita, Phys. Rev. E 65, 026401 (2002).

${ }^{7}$ K. Sawada, K. Eriguchi, and T. Fujimoto, J. Appl. Phys. 73, 8122 (1993).

${ }^{8}$ K. Sawada, thesis, Kyoto University, 1994.

${ }^{9}$ T. Fujimoto, J. Quant. Spectrosc. Radiat. Transf. 21, 439 (1979).

${ }^{10}$ M. Goto, "Collisional-radiative model for neutral helium in plasma revisited," to be published in J. Quant. Spectrosc. Radiat. Transf.

${ }^{11}$ N. N. Ljepojevic, R. J. Hutcheon, and R. W. P. McWhirter, J. Phys. B 17, 3057 (1984).

${ }^{12}$ N. N. Ljepojevic, R. J. Hutcheon, and J. Payne, Comput. Phys. Commun. 44, 157 (1987).

${ }^{13}$ K. Sawada and T. Fujimoto, Phys. Rev. E 49, 5565 (1994).

${ }^{14}$ V. I. Fisher, Y. V. Ralchenko, V. A. Bernshtam, A. Goldgirsh, Y. Maron, L. A. Vainshtein, I. Bray, and H. Golten, Phys. Rev. A 55, 329 (1997).

${ }^{15}$ H. Van Regemorter, Astrophys. J. 136, 906 (1962).

${ }^{16}$ L. A. Vainshtein, I. I. Sobelman, and E. A. Yukov, Electron-Impact Excitation Cross Sections of Atoms and Ions (Nauka, Moscow, 1973).

${ }^{17}$ I. I. Sobelman, L. A. Vainshtein, and E. A. Yukov, Excitation of Atoms and Broadening of Spectral Lines (Springer, New York, 1995).

${ }^{18}$ K. M. Aggarwal, K. A. Berrington, A. E. Kingston, and A. Pathak, J. Phys. B 24, 1757 (1991).

${ }^{19}$ K. M. Aggarwal, J. Callaway, A. E. Kingston, and K. Unnikrishnan, Astrophys. J., Suppl. Ser. 80, 473 (1992).

${ }^{20}$ P. J. Storey and D. G. Hummer, Comput. Phys. Commun. 66, 129 (1991).

${ }^{21}$ T. Fujimoto, J. Phys. Soc. Jpn. 47, 273 (1979).

${ }^{22}$ A. Hirabayashi, Y. Nambu, M. Hasuo, and T. Fujimoto, Phys. Rev. A 37, 77 (1988).

${ }^{23}$ A. Loarte, R. D. Monk, J. R. Martín-Solís et al., Nucl. Fusion 38, 331 (1998).

${ }^{24}$ U. Wenzel, K. Behringer, K. Büchl, A. Herrmann, and K. Schmidtmann, J. Nucl. Mater. 266-269, 1252 (1999).

${ }^{25}$ T. Fujimoto, J. Phys. Soc. Jpn. 54, 2905 (1985). 